\title{
A Scientific Classification of Volatility Models*
}

\author{
Massimiliano Caporin ${ }^{\dagger}$ \\ Dipartimento di Scienze Economiche "Marco Fanno" \\ Università degli Studi di Padova \\ Michael McAleer \\ Department of Quantitative Economics \\ Complutense University of Madrid
}

February 2009

\begin{abstract}
Modeling volatility, or "predictable changes" over time and space in a variable, is crucial in the natural and social sciences. Life can be volatile, and anything that matters, and which changes over time and space, involves volatility. Without volatility, many temporal and spatial variables would simply be constants. Our purpose is to propose a scientific classification of the alternative volatility models and approaches that are available in the literature, following the Linnaean taxonomy. This scientific classification is used because the literature has evolved as a living organism, with the birth of numerous new species of models.
\end{abstract}

\footnotetext{
* The first author gratefully acknowledges the financial support from Italian MUS Grant Cofin2006-13-1140. The second author wishes to thank the Australian Research Council for financial support. This paper was written while the second author was visiting the Dipartimento di Scienze Economiche "Marco Fanno", University of Padova, whose hospita lity and excellent resea rch environment are greatly a ppreciated.

† Corresponding author. Università degli Studi di Padova, Dipartimento di Scienze Economiche "Marco Fanno", Via del Santo, 33, 35123 Padova, email: Massimiliano.caporin@unipd.it
} 


\section{Volatility Classification}

Modeling volatility, or predictable changes over time and space in a variable of interest, is crucial in the natural and social sciences. Life can be volatile, and anything that matters, and which changes over time and space, involves volatility. Volatility seems to have originated in the physical sciences, namely in forecasting extreme pollution events, and has more recently become essential in finance and in any other area in which risk has measurable value and impacts on human life.

Some examples where extreme volatility is relevant are volcanic activity, the occurrence of earthquakes and tsunami, the evolution of weather patterns (such as temperature, wind, rainfall, motion of waves, and solar activity), environmental factors (such as air, water and soil pollution), and financial markets. Without volatility, many temporal and spatial variables would simply be constants. Volatility matters, and hence needs to be specified, estimated, tested and forecasted.

In both the natural and social sciences, analyzing volatility is relevant for forecasting the occurrence of earthquakes, volcanic activity and tsunami, and to measure their implicit risks, to quantity the risk and effects of extreme weather events, to evaluate the evolution of the environment, to hedge (or diversify) financial risks, and to compute capital charges that are required to cover unforeseen and extreme financial market fluctuations. 
Our purpose is to propose a scientific classification of the alternative volatility models and approaches that are available in the literature, following the Linnaean taxonomy. We considered this scientific classification because, over a period of 25 years, this branch of the literature has evolved as a living organism, with the birth of numerous new species of models. Such developments are likely to be of interest to several branches of the social and natural sciences, and may assist researchers to identify the best and most appropriate models for their particular purposes.

In the area of financial econometrics (which could be considered a branch of mathematics and statistics), studies concerned with the analysis of time-varying volatility started in 1982, with the seminal paper on AutoRegressive Conditional Heteroskedasticity (ARCH) models by the 2003 joint Nobel Laureate in Economic Sciences, Engle (1982), which was subsequently Generalized to GARCH in Bollerslev (1986), among others. In recent years, the proposed approaches have literally exploded, leading to an increasing number of generalizations of the original models, so much so that models and acronyms have led to some confusion. It can be difficult to distinguish among the numerous alternative approaches.

\section{Univariate and Multivariate Models}

An important issue for analysis relates to the number of events to be considered, thereby leading to the use of univariate and multivariate models. Univariate models are appropriate for a single event, or where multiple events can be aggregated into a single

event. Multivariate, or portfolio, models are appropriate when the interactions, or 
correlations and covariances, among the multiple events are required to increase the signal, or information, relative to the noise, or random shocks. Multivariate models related to covariances have been considered in the BEKK model of Engle and Kroner (1995), while Engle (2002) considered correlations among multiple events in the DCC model. A comparison of such covariance and correlation models is given in Caporin and McAleer (2008).

For purposes of estimation, testing and forecasting, the statistical properties of the methods are essential in order to draw valid inferences, and to provide sensible interval forecasts. Bayesian methods are used in some case to elicit finite sample properties for cases where computer intensive simulations are necessary, whereas large sample or asymptotic properties are available for a variety of different estimation methods Ling and McAleer (2003).

Volatility can either be latent or observed, depending on the particular specification used, and the data frequency. Latent models, in which the parameters and the latent variables are estimated simultaneously, include conditional volatility models and stochastic volatility models (see Asai et al. (2006) for a review of Multivariate Stochastic Volatility models), and are typically used when high frequency data, such as weekly, daily or hourly, are available. When ultra high frequency data are available, such as data observed by the minute or second, a variety of realized volatility or rangebased models is appropriate (see McAleer and Medeiros (2008) for a review of Realized Volatility models). 


\section{References}

Asai, M., M. McAleer and J. Yu (2006), Multivariate Stochastic Volatility: A Review, Econometric Reviews, 25, 145-175.

Bollerslev, T. (1986), Generalised Autoregressive Conditional Heteroscedasticity, Journal of Econometrics, 31, 307-327.

Caporin, M. and M. McAleer (2008), Scalar BEKK and Indirect DCC, Journal of Forecasting, 27, 537-549.

Engle, R.F. (1982), Autoregressive Conditional Heteroscedasticity with Estimates of the Variance of United Kingdom Inflation, Econometrica, 50, 987-1007.

Engle, R.F. (2002), Dynamic Conditional Correlation: A Simple Class of Multivariate Generalized Autoregressive Conditional Heteroskedasticity Models, Journal of Business and Economic Statistics, 20, 339-350.

Engle, R.F. and K.F. Kroner (1995), Multivariate Simultaneous Generalized ARCH, Econometric Theory, 11, 122-150.

Ling, S. and M. McAleer (2003), Asymptotic Theory for a Vector ARMA-GARCH Model, Econometric Theory, 19, 278-308.

McAleer. M. and M.C. Medeiros (2008), Realized Volatility: A Review, Econometric Reviews, 27, 10-45. 


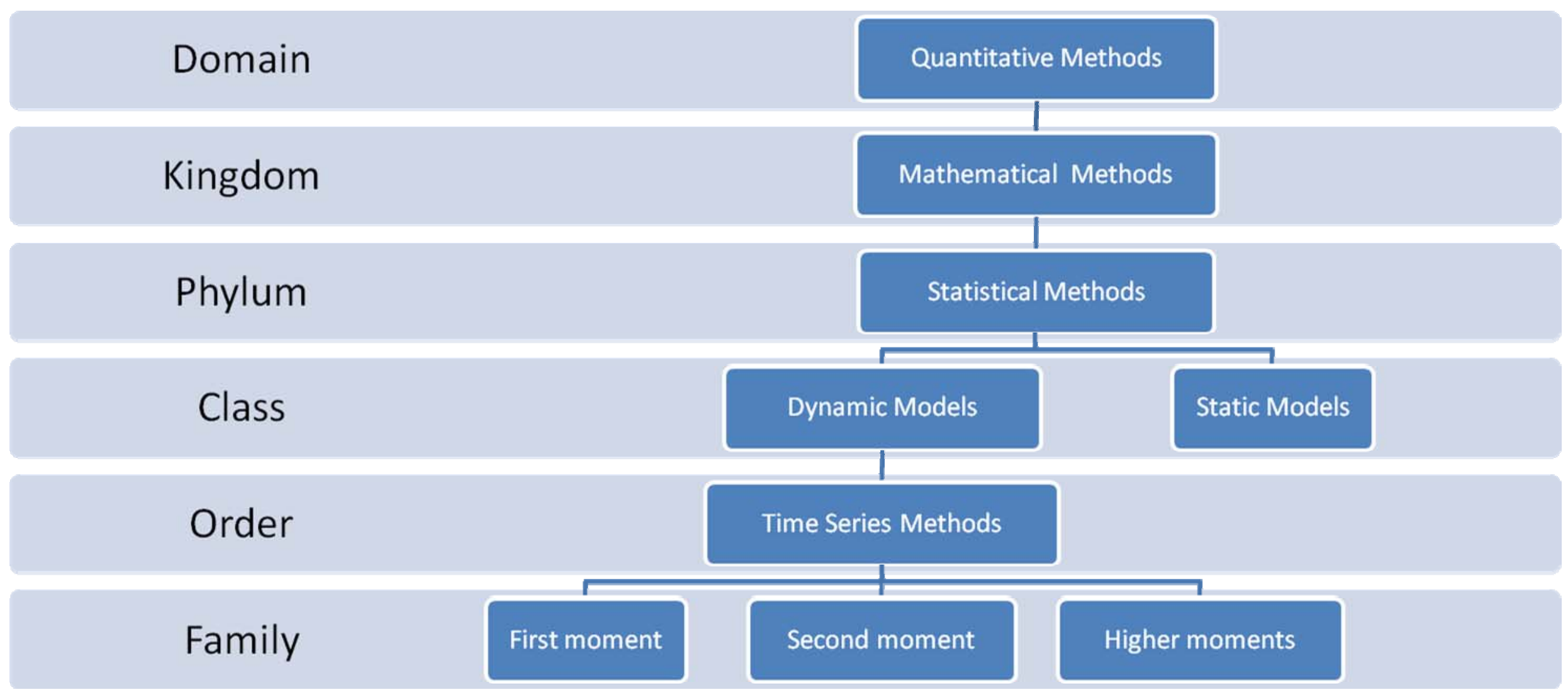

Figure 1 - Panel a) 


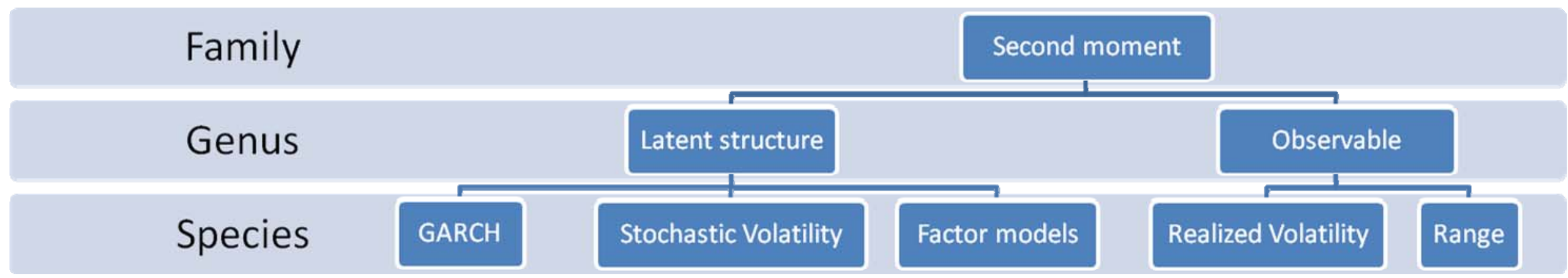

Figure 1 - Panel b)

Figure 1: A Linnaean taxonomy of volatility models from quantitative methods to specific types of volatility models. We define univariate and multivariate specifications as subspecies. 
